\title{
Plexin D1 is a mechanosensor that regulates the site-specific distribution of atherosclerosis
}

ce

PLXND1 is not

just a receptor

for semaphorin

3E, but can

also detect

physical

force and

convert it into

biochemical

signals inside

the cell

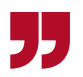

Atherosclerosis is a focal disease.

Areas of arteries with disturbed or

turbulent flow, such as curvatures

or bifurcations, are prone to develop

atherosclerotic lesions, whereas areas

with laminar, uniform flow, such as

straight regions of the vasculature, are

largely protected from the develop-

ment of atherosclerosis. Endothelial

cells in arteries are critical sensors

of flow via mechanoreceptors that

translate the mechanical forces into

intracellular signalling pathways

to induce atheroprotective or ath-

erogenic responses. However, the

mechanoreceptors and signalling

pathways involved in these responses

are incompletely understood. Now,

Ellie Tzima and colleagues describe a

novel mechanoreceptor in endothelial

cells that regulates vascular function

and the site-specific distribution of atherosclerosis. "This study reveals a hitherto unrecognized role for the receptor plexin D1 (PLXND1) in the detection of mechanical force," says Tzima. Plexins are cell-surface receptors for semaphorins and are involved in axonal guidance, tumour progression and immune cell regulation. "We now show that PLXND1 is not just a receptor for semaphorin $3 \mathrm{E}$, but can also detect physical force and convert it into biochemical signals inside the cell that will ultimately dictate the development of atherosclerotic lesions," explains Tzima.

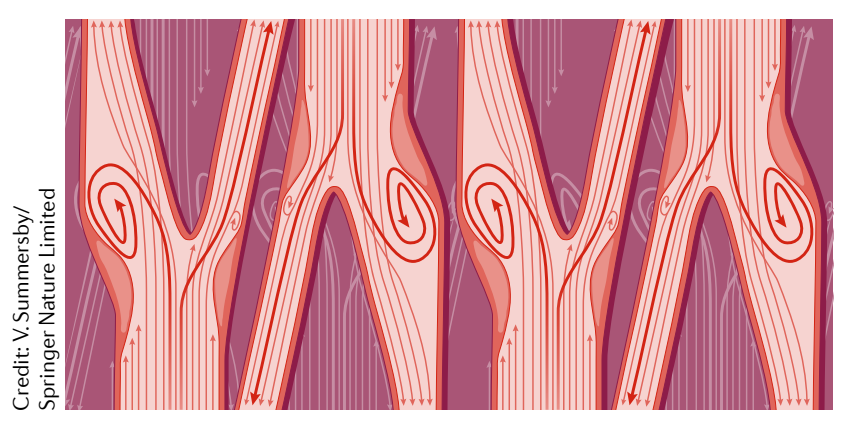

Several endothelial cell mechanosensors have been identified, and one of the best characterized is the PECAM1 junctional mechanosensory complex. "However, what was lacking in these studies was structural information on how the receptors carry out mechanosensation, the relevance in disease and the relationship with other known mechanosensors," comments Tzima. Therefore, the research team set out to address these knowledge gaps.

First, using in vitro and in vivo models, the investigators demonstrate that PLXND1 is required for the response of endothelial cells to shear stress and that the PLXND1-dependent mechanotransduction is independent of its ligand semaphorin 3E. Under laminar-flow conditions, PLXND1 is required for the alignment of endothelial cells in the direction of flow, a hallmark response to atheroprotective shear stress, and the upregulation of Klf2 and Klf4, which encode anti-inflammatory transcription factors. Under disturbed-flow conditions, PLXND1 is involved in the upregulation of pro-inflammatory genes, including Ccl2 and Vcam1.

Next, Tzima and colleagues show that PLXND1 regulates the site-specific distribution of atherosclerotic lesions. In atheroprone mice fed a high-fat diet, PLXND1 deficiency in endothelial cells decreased the plaque burden in the whole aorta and in atheroprone regions (the aortic arch) compared with mice expressing PLXND1 in endothelial cells. PLXND1 deficiency also led to reduced levels of CCL2 and VCAM1 in the inner curvature of the aortic arch.
Finally, the researchers demonstrate that PLXND1 is a direct sensor of force that can elicit mechanical signalling in endothelial cells and they also identify the mechanism by which a single receptor can have a binary function. PLXND1 forms a complex with neuropilin 1 and VEGFR2 in response to flow. This complex acts upstream of the PECAM1 junctional complex and integrins - two mechanosensory hot spots involved in cell-cell and cell-extracellular matrix adhesions, respectively and is sufficient for the response to shear stress. PLXND1 achieves its binary functions as a ligand receptor and a force receptor by switching between two conformational states: a ring-like conformation maintains its ligand-dependent functions, whereas flexion of the ectodomain leads to a more open conformation that is required for mechanotransduction.

“This work not only sheds light on fundamental mechanisms of mechanotransduction that could be applicable to several cell types and organ systems, but also provides answers to the century-old question on the focal distribution of atherosclerosis," says Tzima. Tzima and colleagues are currently screening small-molecule libraries to identify inhibitors of PLXND1 and assess whether they have antiatherogenic effects. "The idea would be to repurpose known drugs to target PLXND1 signalling for the prevention of atherosclerosis," explains Tzima.

Irene Fernández-Ruiz

ORIGINAL ARTICLE Mehta, V. et al. The guidance receptor plexin D1 is a mechanosensor in endothelial cells. Nature 578, 290-295 (2020) RELATED ARTICLE Souilhol, C. et al. Endothelial responses to shear stress in atherosclerosis: a novel role for developmental genes. Nat. Rev. Cardiol. 17, 52-63 (2020) 